\title{
El recurso cuprífero de Cerro Colorado, Comarca Ngäbe-Buglé, Panamá (1946-2014). Factor de negociación o de represión entre el Estado panameño y el grupo ngäbe
}

\section{The Cerro Colorado Copper Resource, Ngäbe-Bugle, Panama (1946-2014). Bargaining Chip or Means of Repression between the Panamanian Government and the Ngäbe Group}

\author{
Jorge Luis Roquebert León \\ Doctorado en Humanidades y Ciencias Sociales \\ Universidad de Panamá, Panamá | jlroquebert.leon@gmail.com
}

\begin{abstract}
Resumen
El artículo forma parte de una investigación más amplia sobre la relación del Estado panameño y el grupo ngäbe a partir de la segunda mitad del siglo $\mathrm{xx}$ hasta el presente. En alguna medida, busca estudiar el manejo de los recursos minerales del país, enfocándose en los existentes en la Comarca Ngäbe-Buglé, creada en 1997. Deja expuesto que el ánimo que ha movido las promulgaciones de las leyes, a través del periodo de estudio, no ha sido el mismo y ha generado reacciones en los grupos involucrados, repercutiendo en diferentes aspectos de la gobernabilidad y gobernanza en el país.
\end{abstract}

Palabras claves: Comarca Ngäbe-Buglé; leyes de recursos minerales; yacimientos de cobre; gobernabilidad; gobernanza.

\begin{abstract}
The article is part of a broader study on the relationship between the Panamanian government and the Ngäbe group from the second half of the 20th century to the present. The author seeks to study the management of mineral resources in the country, focusing on those in the Ngäbe-Bugle created in 1997. It shows that the spirit that has driven the enactments of laws, throughout the period under review, has not been the same and has elicited reactions among the groups involved, affecting different aspects of governability and governance in the country.
\end{abstract}

Key words: Ngäbe-Bugle Province; mineral resource laws; copper deposits; governability; governance.

Fecha de recepción: 18 de noviembre de 2014 Fecha de aceptación: 22 de abril de 2015

Secuencia, ISSN 0186-0348, núm. 96 | septiembre-diciembre de 2016 | pp. 167-192 


\section{El recurso cuprífero de Cerro Colorado, Comarca Ngäbe-Buglé, Panamá (1946-2014). Factor de negociación o de represión entre el Estado panameño y el grupo ngäbe} Jorge Luis Roquebert León

\section{INTRODUCCIÓN}

Zl yacimiento de cobre en Cerro Colorado, Comarca Ngäbe-Buglé, en la Erepública de Panamá, es uno de los mayores a nivel mundial, con capacidad de explotación de hasta 100 años o más. Su exploración comenzó a mitad del siglo pasado, pero no fue hasta la década de 1970 en que el Estado creó la Corporación de Desarrollo Minero (CODEMIN), como concesionaria para su exploración y explotación. Sin embargo, al no poseer el capital y la tecnología para realizar ambas tareas, se hacía necesario que el Estado incorporase a compañías mineras multinacionales como estrategia para la administración del proyecto.

En este trabajo se compara la gobernabilidad y la gobernanza en los periodos de estudio y su influencia en la visión de los gobiernos, en las normativas que crean para la explotación del depósito de cobre, y en la preparación de los instrumentos legales, teniendo presente que la ubicación de este recurso mineral se encuentra en territorio habitado por la población ngäbe y buglé, ${ }^{1}$ lo que imprime un tratamiento diferente debido a que la promulgación de la ley creadora de su comarca buscaba el establecimiento de un ambiente favorable a la gobernabilidad, tal como la participación ciudadana de los ngäbes, la búsqueda de un consenso previo a la realización de proyectos en su comar-

${ }^{1}$ El grupo ngäbe forma parte de la familia lingüística macrochibcha y en la actualidad es el grupo indígena más numeroso de la República de Panamá. Su historia en el territorio panameño se remonta al periodo prehispánico, cuando sus límites territoriales se extendían desde la actual provincia de Coclé hasta el sur de Costa Rica. El grupo buglé está ligado también a la misma familia lingüística, si bien su idioma, el buglere, es ininteligible con el ngäbere de los ngäbes. Ambos grupos coexisten y habitan la región occidental de Panamá y lograron la aprobación de una comarca por parte del Estado en 1997.

\section{()ㅜ(1) $\$$}


ca y sobre todo la equidad de etnias, para así lograr finalmente el ambiente de estabilidad necesario para una gobernanza exitosa que desarrollara la relación de los grupos ngäbe y buglé, su comarca y el Estado panameño.

Para lograr los objetivos de la investigación, se divide el estudio en tres periodos históricos, en donde las orientaciones dictadas por los organismos internacionales se reflejan en los patrones de gobierno en el ámbito nacional. Esto tiene una consecuencia en el tema de la minería, por el tipo de mercado en el que funciona. Estos tres periodos son de 1946 a 1968, de 1968 a 1989, y de 1990 a 2014.

\section{CARACTERIZACIÓN DE LOS TRES PERIODOS}

\section{Primer periodo -1946 a 1968}

La aprobación de una nueva Constitución en 1946, influenciada por las ideas antirracistas del tiempo de la posguerra, reservaba a las colectividades campesinas e indígenas el capítulo 6 del título III, Derechos individuales y sociales, por el cual el Estado se comprometía con los dos grupos a integrarlos a la comunidad nacional "en cuanto a sus normas de vida, lo económico, lo político y lo intelectual. La acción relativa a los indígenas se efectuará conservando y desarrollando al mismo tiempo los valores de la cultura autóctona" (artículo 94). Sin embargo, las leyes promulgadas no mostraron desarrollar el espíritu de este compromiso, dando margen a rebeliones por parte de los grupos que buscaban ser protegidos en la Constitución debido a que la visión que en la práctica se aplicó, como por ejemplo en lo referente a la educación y la reforma agraria, fue una de homogenización de los grupos por parte del Estado, atentando contra la preservación y el respeto a las culturas indígenas.

En el plano nacional, de 1946 en adelante se refleja la crisis económica internacional que marcó la finalización de la guerra mundial, por lo que para las clases gobernantes panameñas se hacía necesaria la renegociación del tratado que facilitara un mejor acceso económico a los beneficios de la Zona del Canal, administrada como territorio estadunidense desde los tratados iniciales entre ambos países de 1903. La recuperación económica fue lenta, y la negociación del nuevo tratado con Estados Unidos se logró en 1955. Sin embargo, a partir de 1960 comenzó una lenta industrialización en el país, que se vio obstaculizada por las peculiaridades locales, como los recursos y el mercado limitado y la dependencia económica.

\section{(1)(1) $\$$}


Gandásegui (2008) también explica que la situación política no fue estable, sobre todo en los periodos presidenciales de 1948 y 1952, debido a los conflictos entre los partidos gobernantes y los de oposición, por la presión del poder de la Guardia Nacional, ${ }^{2}$ unido al resentimiento creciente por parte de la población en contra de la presencia estadunidense en la zona de tránsito, ${ }^{3}$ que tuvo su punto de detonación con los eventos del 9 de enero de 1964, en el cual un grupo de estudiantes del Instituto Nacional de Panamá murieron, luego de ser atacados por la población civil y el ejército estadunidense acantonado en la Zona del Canal que se oponían al mandato federal de izar la bandera panameña junto a la de Estados Unidos, provocando el rompimiento de relaciones diplomáticas de Panamá con este país.

\section{Segundo periodo -1968 a 1989}

En el plano político, este periodo es la culminación del anterior, cuando el gobernante electo en 1968 intenta controlar a la Guardia Nacional, situación que conlleva su derrocamiento y la prohibición de la democracia partidaria. Este evento violentó la Constitución de 1946, pero con la aprobación de una nueva carta fundamental en 1972, el grupo militar gobernante legitima una nueva forma de gobernabilidad.

Durante la década de 1970 el Estado panameño retomó la lucha contra el enclave estadunidense en la zona de tránsito de su territorio y logró

2 Se refiere a que en los ocho años entre 1948 y 1956 hubo siete presidentes, y no fue sino hasta el "régimen fuerte" del jefe de la Policía Nacional, José Remón (1952-1955), que Panamá volvió a su tradicional sistema de "libre competencia". Sin embargo, cuando fue asesinado, su vicepresidente fue procesado por la Asamblea Nacional acusado del magnicidio. En los periodos de 1956, 1960 y 1964 se sucedieron en la presidencia de la república Ernesto de la Guardia, Roberto Chiari y Marco Robles, siendo los dos primeros primos de Remón y de Robles de Chiari. También explica que si bien el presidente Chiari (1960-1964) es recordado por su posición patriótica al enfrentarse al gobierno de Estados Unidos por los incidentes del 9 de enero, su gobierno en su momento era calificado como impopular y sus acciones dirigidas al enriquecimiento de una clase sustentadora.

${ }^{3}$ Por la zona de tránsito en Panamá se entiende la ruta que históricamente ha sido utilizado para el comercio posterior a la colonización española. Durante los siglos XIX y XX, paralelo a la construcción del ferrocarril transístmico en 1855 y a la construcción del canal en 1914, los puertos de entrada han sido la ciudad de Panamá en el océano Pacífico y la ciudad de Colón en el mar Caribe. Durante el periodo colonial el puerto de entrada en el mar Caribe estaba en Nombre de Dios (siglo XVI) y Portobelo (siglos XVI al xIX) y se utilizaba el llamado Camino de Cruces para la movilización de mercancías entre España y el virreinato de Perú.

\section{()(1) $\$$}




\section{SECUENCIA}

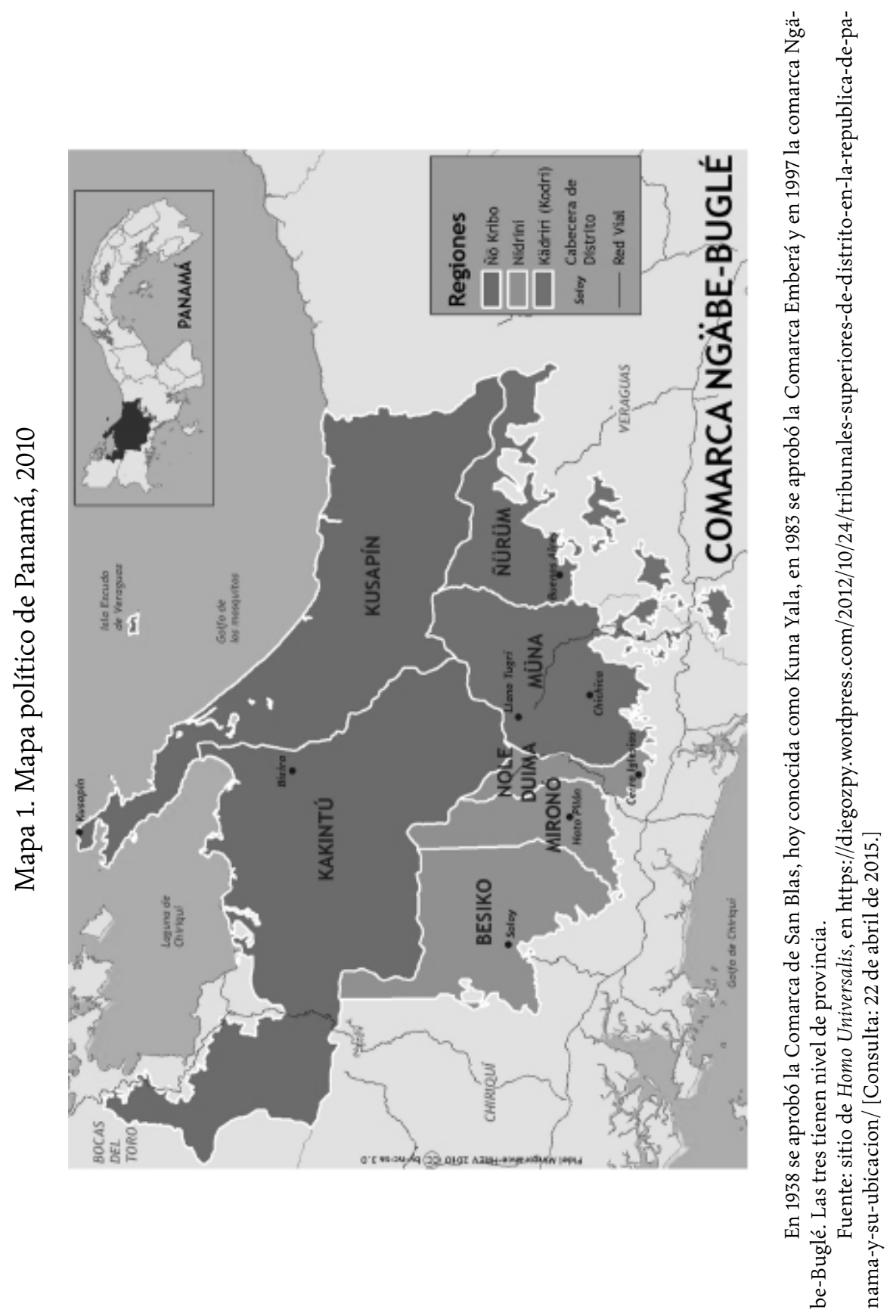

\section{(ㅇ)(1) 8}


firmar en 1977 un nuevo tratado con Estados Unidos por el que se acordaba finalizar el territorio de la Zona del Canal en 1979; a partir de este año habría una administración conjunta en la compañía canalera, que continuaría funcionando como agencia federal de Estados Unidos, pero su junta directiva estaría integrada por estadunidenses y panameños hasta finales del siglo xx, cuando volvería en su totalidad al Estado panameño.

Si ocurrió un enfrentamiento entre los gobiernos panameño y estadunidense por la posesión de la zona de tránsito, en esta década el gobierno panameño también encaró a la compañía bananera estadunidense, United Brands Company, que opera en la zona agropecuaria al occidente de la república, en las provincias de Chiriquí y Bocas del Toro. Manfredo (1976) explica que no sólo se logró, junto a otros países del área, establecer un impuesto a la compañía por las cajas de banano exportadas, sino un nuevo contrato más favorable para la nación panameña.

Tanto Soler (2008) como Manduley (2014) explican cómo la economía internacional también influyó en la nacional durante este periodo, ocurriendo una industrialización impulsada desde el Estado durante la década de 1970 movida con empréstitos de la banca internacional. Además, Manduley (2014) detalla que los objetivos de la política económica del nuevo régimen se concentrarían en la ampliación de las operaciones de la Zona Libre de Colón, la conversión del aeropuerto de Tocumen (ciudad de Panamá) en centro de producción y reventa de bienes y servicios, el turismo, la explotación de recursos minerales, la promoción de Panamá como centro financiero internacional y la continuación de la política de sustitución de importaciones.

En la década de 1980, por el contrario, siguiendo las políticas neoliberales impulsadas desde el exterior, el papel del Estado contrajo su participación en la economía. Además, la política exterior con el gobierno de Estados Unidos se vio afectada, sobre todo a partir de 1987, cuando las Fuerzas de Defensa, otrora Guardia Nacional, entraron en conflicto abierto con Estados Unidos, lo que concluyó con una invasión militar el 20 de diciembre de 1989.

Tercer periodo -1990 a 2014

Como consecuencia de la invasión y la destrucción de las Fuerzas de Defensa, en esta fase quedó descartada la presión ejercida por la institución castrense sobre los gobiernos, como sucedió en los dos periodos precedentes. Aunque en mayo de 1989 salió triunfadora la alianza política contraria a las

\section{()(1) $(9$}




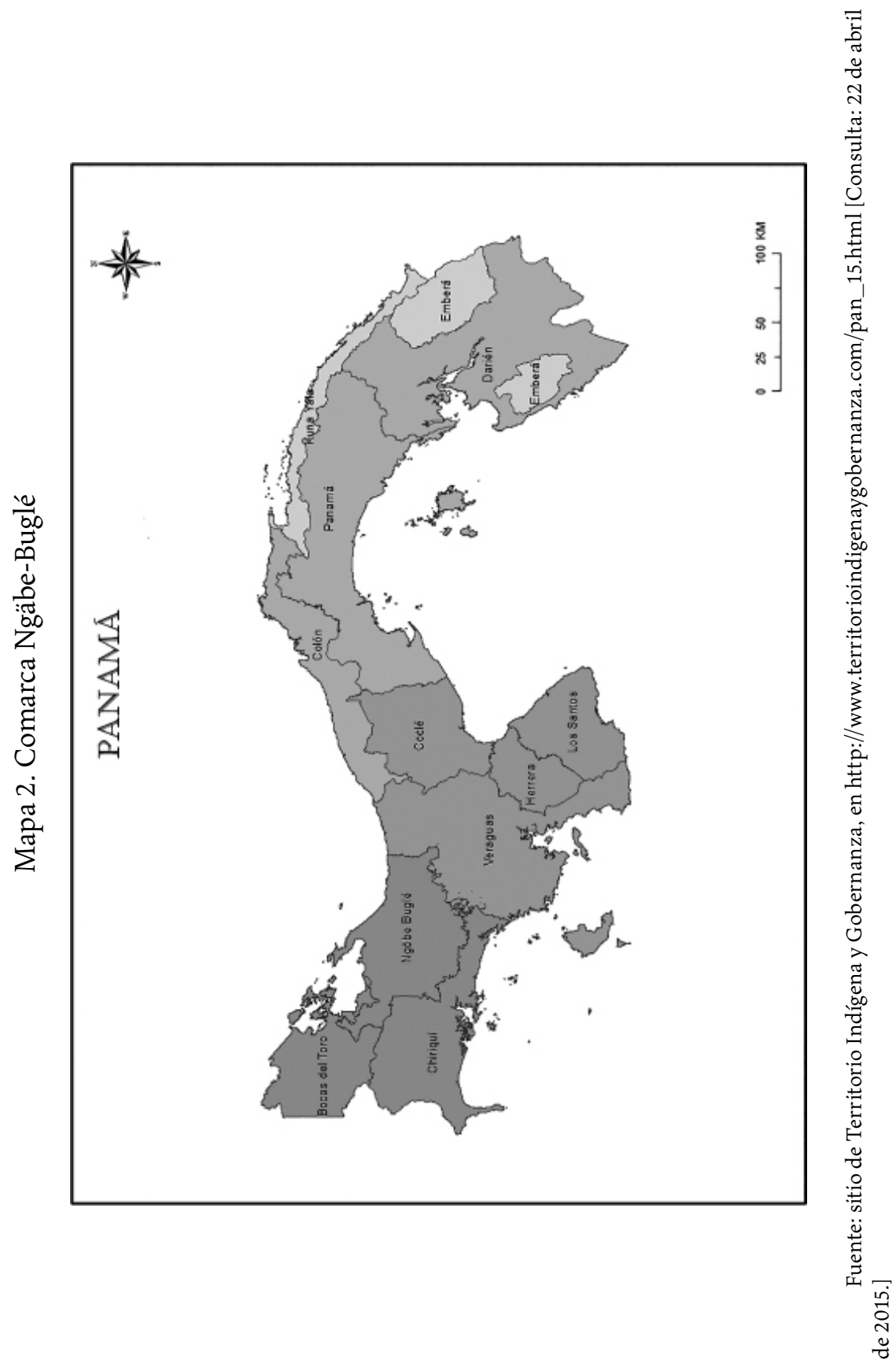

\section{(ㄷ)(1) (8)}


fuerzas armadas, para la siguiente elección nacional, en 1994, el partido político que apoyó al régimen militar de 1968 a 1989, el Partido Revolucionario Democrático (PRD), regresó al poder, y ha estado a cargo del gobierno de manera alternativa en los siguientes periodos electorales con el otro partido más importante en cuanto a porcentaje de población, el Partido Panameñista.

En el plano económico se completan las reformas neoliberales iniciadas a partir de 1981 por el PRD y continuadas por los partidos que lo han sucedido en el manejo de la cosa pública, demostrando que el enfoque económico no cambia con los gobiernos. Y aunque el crecimiento de la economía nacional se ha mostrado como una fortaleza de los gobiernos, incluso en el quinquenio 2009-2014, que está marcado por una crisis mundial a partir de 2008, la desigualdad en la distribución de la riqueza en la sociedad panameña se manifiesta como una debilidad, razón que explica la insatisfacción ciudadana, que se refleja en disturbios populares de niveles alarmantes, mas no duraderos, ante la propuesta del gobierno que inició en 2009 de restringir los beneficios laborales, como sucedió con la Ley 30 del 16 de junio de 2010, que buscaba introducir, entre otras cosas, cambios al Código de Trabajo, modificando el derecho a huelga de los trabajadores y eliminando el descuento obligatorio de la cuota sindical que reciben los sindicatos del país. ${ }^{4}$

El manejo de la insatisfacción de la población, que busca una participación más activa en la economía y la política del país, puede ser el indicio de la entrada a un nuevo periodo marcado por una mayor integración de los diferentes grupos que forman la nación panameña, o de represión por parte del grupo gobernante.

\section{CRONOLOGÍA DE LA LEGISLACIÓN MINERA APLICABLE A CERRO COLORADO}

El punto de partida para estudiar algún tema minero en Panamá está en el articulado de las Constituciones (1946 y 1972) y en el Código de Recursos

${ }^{4}$ La Ley 30 de 2010, conocida popularmente como "Ley 9 en 1" o "Ley Chorizo", fue presentada como una ley de aviación comercial, pero reformó tres códigos y seis leyes, cambiando los procedimientos en materia ambiental, laboral, social y policial. A pesar de las protestas de grupos ambientalistas y sindicales en la ciudad de Panamá, su mayor impacto se produjo en la provincia de Bocas del Toro, en donde una huelga de los empleados de la empresa bananera llevó a conflictos con la fuerza armada que produjeron muertos y heridos, y el sitio de Changuinola. Véase Changuinola en Caos. La Prensa, 8 de julio de 2010.

\section{()(1) $\$$}


Minerales (1963) que, siguiendo la tradición hispana, establecen que el Estado es propietario del subsuelo. ${ }^{5}$ Así, se tiene que la Constitución de 1946 establece en su artículo 208 que pertenecen al Estado "5) El subsuelo, que puede ser objeto de concesiones para la explotación de sus riquezas según lo establezca la Ley".

El Código de Recursos Minerales, publicado como Decreto Ley del 22 de agosto de 1963, tiene importancia hasta la actualidad, porque no sólo establece los derechos del Estado y del concesionario, sino porque establece claramente quiénes no pueden beneficiarse de estas concesiones,

Derechos de Propiedad del Estado, Artículo 2: Los yacimientos minerales de toda clase existentes en todo el territorio de la República de Panamá [...] son de propiedad del Estado, [...] Los minerales extraídos mediante concesiones mineras otorgadas de conformidad con este Código pertenecen al concesionario.

Título II, Otorgamiento de concesiones mineras, Artículo 4: No podrán obtener concesiones mineras por sí, ni por interpuesta persona, ni podrán ejercerlas o disfrutarlas, ninguno de los que a continuación se mencionan: Los Gobiernos o Estados Extranjeros, ni ninguna entidad o institución oficial o semioficial extranjera, ni las personas jurídicas en las cuales tenga participación directa o indirecta algún gobierno o Estado extranjero. ${ }^{6}$

La Constitución de 1972, a diferencia de la Constitución de 1946 que limitaba a concesiones privadas la explotación de los recursos minerales, es más abarcadora en el derecho que guarda el Estado, y establece en su artículo 257 lo siguiente: pertenece al Estado "5. Las riquezas del subsuelo, que podrán ser explotadas por empresas estatales o mixtas o ser objeto de concesiones o contratos para su explotación según lo establezca la Ley. Los derechos mineros otorgados y no ejercidos dentro del término y condiciones que fije la Ley, revertirán al Estado."

${ }^{5}$ Mena García (2011, p. 369) explica cómo la tradición minera hispana que se heredó de tiempos de la colonia descansa en el principio planteado en las Siete Partidas, de Alfonso X el Sabio, sobre la propiedad del subsuelo. Sin embargo, cita a Ots Capdequí que afirma que una de las dificultades al hacer las reconstrucciones hasta 1680 fue que los derechos concedidos a los descubridores de minas podían variar en casi todo momento.

${ }^{6}$ Hay que tener presente la restricción planteada por este artículo, ya que guarda importancia en el desarrollo de eventos recientes de la gobernabilidad y la gobernanza minera de nuestro país.

\section{()(1) $\$$}


Esta ampliación no sólo guarda relación con el proyecto de la explotación minera en el territorio de la actual Comarca Ngäbe-Buglé, en la década de 1970, sino que responde a experiencias de nacionalización de recursos en la región latinoamericana, de las cuales el mejor ejemplo es el caso chileno: la creación de la Corporación del Cobre (CoDelco) en 1971 fue el evento final de las legislaturas comenzadas en la década de 1950, que llevaron a la llamada "chilenización del cobre" en la de $1960 .^{7}$

El conocimiento de la existencia de venas mineralizadas de cobre en Cerro Colorado data de 1930. En 1955 el Estado panameño, asistido por la Compañía del Canal de Panamá y las Naciones Unidas, comenzó a realizar exploraciones en el área. Con base en estos descubrimientos, durante la primera mitad del decenio de 1970 el Estado realizó mayores exploraciones y, por medio de la Ley 41 de 1975, creó la empresa estatal CoDEMin, adscrita al Ministerio de Comercio e Industrias, que sería la encargada de administrar y explotar "los productos derivados y sustancias asociadas del yacimiento minero de Cerro Colorado, así como la realización de actividades relacionadas con proyectos especiales que le asigne el Órgano Ejecutivo" (artículo 2). La CoDEmin se encargaría de crear la sociedad anónima Empresa de Cobre Cerro Colorado, S. A., y entre su fecha de creación y 1981, realizaría contratos para compartir acciones con empresas mineras multinacionales para la exploración y explotación del recurso cuprífero (Gjording, 1991, pp. 77-78). ${ }^{8}$

Sin embargo, la conjunción de situaciones internacionales y nacionales influyó en el desenlace del proyecto minero. Entre las primeras, la más importante fue la baja del precio del cobre en el mercado internacional, mientras que entre las segundas estuvieron el cambio de gobernantes a partir

\footnotetext{
${ }^{7}$ Las consecuencias políticas sufridas por este país a causa de contradecir los intereses de las compañías multinacionales son conocidas. Cuadra (2013) se refiere al informe Church, elaborado por el Senado estadunidense en 1975, y explica cómo la desestabilización del gobierno de Salvador Allende fue financiada por la cia desde antes de que asumiera la presidencia en 1970, al ser visto como un serio riesgo y por la amenaza que representaba el convertir a Chile en una "segunda Cuba" en América Latina. Ventura (2013) explica que Allende intentó instaurar un socialismo por la vía pacífica y constitucional en su país, un experimento que fue visto no sólo en América Latina, sino en el resto del mundo; su modelo económico socialista en Chile fue remplazado por otro nuevo, el neoliberal o neoconservador, incluso antes de que se intentara implementar en otras naciones de Occidente.

${ }^{8}$ Las empresas mineras multinacionales serían la canadiense Javelin, Ltd., la americana-canadiense Texasgulf, Inc, y la británica Rio Tinto Zinc (RTz). En Geología de Azuero, se presenta el número de acciones compartidas por las dos primeras empresas, y es importante que para 1995, luego del estancamiento en las negociaciones, Panamá tenía de vuelta 100\% de las mismas.
}

\section{()(1) $(3$}


de la muerte del general Omar Torrijos, el mayor propulsor de la realización de la obra en Cerro Colorado, y la oposición al mismo de sectores que temían que un gobierno que hubiese recuperado la Zona del Canal para Panamá y lograra después traer al país los beneficios económicos que la explotación minera implicaba, sería imposible de vencer en unas elecciones partidistas, las cuales fueron anunciadas una vez firmado el tratado de 1977 (Gjording, 1991, pp. 141-147). ${ }^{9}$ Esto provocó que los accionistas de la Empresa de Cobre Cerro Colorado, S. A. -la estatal CoDEMIN y la británica RTZ- resolvieran en 1981 entrar en una etapa de cuido y mantenimiento, y si pasado el tiempo acordado el proyecto no iniciaba, la concesión de Cerro Colorado regresaría totalmente al Estado panameño. ${ }^{10}$

El tema del cobre en Cerro Colorado fue dejado en segundo plano por los gobiernos, pero después de 1985 se sancionaron leyes mineras que modificaron el Código de Recursos Minerales que tienen repercusiones en la relación entre el Estado y las comunidades nacionales. Así, la Ley 3 del 28 de enero de 1988, en su artículo 20, establece:

A los municipios de las provincias y comarcas donde se establezcan instalaciones de extracción minera con posterioridad a la entrada en vigor de la presente Ley, les corresponderá el quince por ciento $(15 \%)$ de los beneficios que perciba el Estado de tales actividades, suma que será destinada a la realización de programas de desarrollo, obras y/o servicios en los respectivos distritos, los que serán elaborados por el Ministerio de Planificación y Política Económica, dentro de un orden de prioridades aprobadas por la Asamblea Legislativa.

Además, mediante el artículo 8 de esta ley, se modificó el artículo 41 del Código de Recursos Minerales, estableciéndose una nueva clasificación a los minerales: minerales no metálicos; minerales metálicos excepto los minerales preciosos; minerales preciosos aluvionales; minerales preciosos no aluvionales; minerales energéticos, excepto los hidrocarburos, y minerales de reserva. La clasificación previa en el Código de Recursos Minerales de 1963 hacía referencia a la profundidad en que se encontraban los metales, y

${ }^{9}$ Como se explicará más adelante, en el punto de gobernabilidad territorial, esta decisión pudo afectar también la negociación de la comarca con los ngäbes y los buglés en 1983.

${ }^{10}$ Luque, V. (7 de julio de 2014). Entrevista con el profesor en Derecho Minero Virgilio Luque/Entrevistador: Jorge Luis Roquebert León. Universidad de Panamá, Panamá.

\section{()(1) $\$$}


la mención de los hidrocarburos en la modificación al Código de Recursos Minerales se debe a que hasta el año anterior, cuando se aprobó la Ley 8 del 16 de junio de 1987, estos se habían regulado por el código debido a su composición química.

Sin embargo, al igual que a comienzos de la década de 1980 el mercado mundial fue un factor que influyó en la toma de decisión para que el proyecto entrara en una etapa de cuido y mantenimiento, desde la década de 1990 surgen nuevos clientes de cobre en Asia y se da un alza en los precios del mismo que renovó el interés del Estado y de las compañías mineras multinacionales para explotar los yacimientos cupríferos del país. En 1997 se otorgaron concesiones en el distrito de Donoso, provincia de Colón, a compañías en las que participaban ciudadanos panameños allegados al gobierno nacional junto a compañías mineras canadienses como accionistas. ${ }^{11}$

Desde finales de 2009 una empresa de Corea del Sur se mostró interesada en participar en el proyecto Cobre Panamá concesionado a Minera Panamá, S. A., propiedad de la compañía canadiense Inmet, ubicado en el distrito de Donoso. ${ }^{12}$ En una visita a ese país, el presidente panameño aceptó revisar la ley minera panameña para que pudieran participar en los proyectos de recursos minerales. ${ }^{13}$ La revisión del Código de Recursos Minerales de 1963 era necesaria para hacer posible la participación de la empresa surcoreana interesada, ya que permitir al Estado surcoreano tener acciones en esta violaría el artículo $4^{\circ}$ de dicho código, que establecía la prohibición a las em-

${ }^{11}$ Los contratos de Richard Fifer. La Prensa, 31 de octubre de 2007; Grupo de Fifer recibió millones. La Prensa, 29 de septiembre de 2008; Minera alega tener 1.4\% de todo el país. La Prensa, 6 de mayo de 2013; Petaquilla, fuera de sus límites. La Prensa, 7 de mayo de 2013.

${ }_{12}$ Panama: Korean Mining Company Will Invest in Copper Project, 30 de octubre de 2009. CentralAmericaData.com. Business Information. Recuperado de http://www. centralamericadata.com/en/article/home/Panama_Korean_Mining_Company_Will_Inve st_in_Copper_Project

${ }^{13}$ En el primer cuatrimestre de este año, el presidente surcoreano realizaba una visita diplomática a Panamá, la primera reunión bilateral de presidentes de los dos países desde el establecimiento de relaciones en 1962, y se anunciaba que el presidente Martinelli ofrecía Cerro Colorado a los coreanos, anunciando ante un grupo de inversionistas que cambiaría la legislación minera a solicitud del gobierno surcoreano para explotar el yacimiento de cobre de Cerro Colorado, en la Comarca Ngäbe-Buglé. Martinelli ofrece Cerro Colorado a los coreanos. La Prensa, 26 de marzo de 2010. Más adelante, en una visita del presidente panameño a Corea del Sur, un portavoz del gobierno de Seúl comunicó que "Martinelli expresó que las empresas surcoreanas podrán participar en la ampliación del Canal y 'prometió' revisar la ley minera para que puedan estar en proyectos de recursos mineros." Presidente de Corea hablará en Panamá sobre la minería. Panamá América, 20 de abril de 2010.

\section{(ㄷ)(1) $(3$}


presas en las que algún gobierno o Estado extranjero tuviese participación, de ejercer o disfrutar concesiones mineras.

La Ley 8 del 11 de febrero de 2011, la cual reforma el Código de Recursos Minerales y dicta otras disposiciones relacionadas con la actividad minera, buscaba solucionar este impedimento a la empresa surcoreana al establecer en su artículo $4^{\circ}$ :

No podrán obtener concesiones mineras, ni ejercerlas o disfrutarlas los siguientes entes o personas:

1. Los gobiernos o Estados extranjeros o instituciones oficiales o semioficiales extranjeras, con excepción de las personas jurídicas en las que tengan participación económica o financiera uno o más estados o gobiernos extranjeros o instituciones oficiales o semioficiales extranjeras, siempre que dichas personas estén constituidas como personas jurídicas de Derecho Privado bajo las normas panameñas, renuncien expresamente en el contrato de concesión a la reclamación por vía diplomática, salvo el caso de denegación de justicia, y se sometan en el contrato a las leyes de la República de Panamá.

Esta situación llevó a serias protestas de la población ngäbe y buglé, especialmente en su comarca, por sentirse en riesgo de perder los logros obtenidos, si bien el artículo 29 de la ley -al igual que el artículo 48 de la Ley 10 de 1997, por la cual se crea la Comarca Ngäbe-Buglé- dejaba claramente estipulado que el Estado y el concesionario debían realizar una consulta previa con "un plan de divulgación para que las autoridades y las comunidades indígenas sean informadas y puedan plantear voluntariamente su punto de vista respecto a la actividad minera; además, deberán garantizar los derechos de la población en beneficio y cumplimiento de los principios de desarrollo sostenible y protección económica, procurando su participación de conformidad con las leyes que crean dichas comarcas."

La oposición a la nueva ley por parte de los grupos ngäbe y buglé se dio a pesar de los beneficios económicos que el gobierno central le otorgaba a los municipios y comarcas: el artículo 26 de la Ley 8 de 2011 modificaba los beneficios otorgados en 1988 subiéndolos de 15 a 20\%, de los cuales 5\% serían distribuidos entre los corregimientos colindantes al proyecto.

El artículo 20 de la Ley 3 de 1988 quedó así:

A los municipios y comarcas donde se realicen actividades de exploración y explotación minera por razón de una concesión, les corresponderá el 20\% de

\section{(ㅇ)(1) $\$$}


los beneficios que, de acuerdo con el Código de Recursos Minerales, deba percibir el Estado de tales actividades. Dicho ingreso será recaudado directamente por estos municipios y comarcas, con base en los cálculos que prepare la Dirección Nacional de Recursos Minerales del Ministerio de Comercio e Industrias, y será dirigido únicamente a programas de desarrollo en áreas de educación, salud y proyectos de inversión social requeridos por las comunidades.

Del porcentaje establecido en este artículo, corresponderá el 5\% a los corregimientos colindantes con los municipios y comarcas en un radio de distancia de hasta 35 kilómetros de las concesiones donde se realicen actividades de exploración y explotación minera por razón de una concesión. Este ingreso será transferido directamente a las juntas comunales o comarcas respectivas que lo distribuirán equitativamente entre estas, exclusivamente para programas de desarrollo en áreas de educación, electrificación rural, salud y proyectos de inversión social requeridos por las comunidades.

Después de tres días de protestas convocadas por la Coordinadora por la Defensa de los Recursos Naturales y el Derecho del Pueblo Ngäbe Buglé y Campesinos en contra de la reforma del Código de Recursos Minerales, el 26 de febrero la policía enfrentó a los manifestantes con un saldo de al menos doce indígenas heridos, quince policías golpeados, y una bebé de dos meses y dos mujeres afectadas por los gases lacrimógenos. Sin embargo, para el final del siguiente día el gobierno y los indígenas lograron un acuerdo de seis puntos: 1. La instalación de una comisión de alto nivel, integrada por representantes del gobierno y de la Coordinadora. 2. La creación de una ley que prohibiese explícitamente la minería en la comarca y protegiese los recursos hídricos. 3. Que se diera inicio al diálogo el 1 de marzo para analizar cada artículo de la Ley 8 de 2011. 4. La liberación de todos los detenidos, la no represalia contra los dirigentes de la Coordinadora y el apoyo por parte del gobierno a los afectados de los disturbios. 5. La designación de la Iglesia católica como mediadora y garante del diálogo. 6. La reapertura inmediata de todas las vías, en especial de la vía Interamericana. ${ }^{14}$ Ante la magnitud de las protestas, el Estado derogó la Ley 8 de 2011, por medio de la Ley 12 del 18 de marzo de 2011.

$\mathrm{Al}$ año siguiente, ante el fracaso para llegar a un consenso con el Estado, el grupo indígena cerró la vía Interamericana como medida de presión, ocurriendo hechos de violencia entre los miembros de la Coordinadora y la

${ }^{14}$ Indígenas y gobierno acuerdan despejar vías. La Prensa, 28 de febrero de 2011.

\section{(ㄷ)(1) $(3$}


policía, con resultado de un muerto y varios heridos entre los manifestantes. José Luis Lacunza, obispo de la diócesis de David, provincia de Chiriquí, y mediador en los conflictos entre el gobierno y los indígenas, explicó que el origen del nuevo conflicto se encontraba en la lentitud en las negociaciones, tanto que para finales del año no habían logrado la elaboración de una nueva ley, tal como había sido pactado, en la Comisión de Comercio de la Asamblea Nacional, y al momento de presentarla se hizo "sin el artículo quinto en el que los indígenas pedían se cancelaran los proyectos hidroeléctricos en la comarca y áreas anexas", por lo que comenzó primero el cierre parcial de las calles y luego de manera total. Para la solución del problema declaraba que las partes debían sentarse a resolver un conflicto de vieja data. "En 1997 nos quedamos tranquilos porque les dimos la comarca y iqué hemos hecho con eso? Encerrarlos allí para que se nos mueran de hambre. No hay un plan de desarrollo integral de la comarca. Ese era uno de los puntos que el año pasado se acordó, que cada ministro iba a presentar un plan de desarrollo de la comarca. Promesas y promesas que se hacen". ${ }^{15}$

Además, en medio de los conflictos el gobierno procedió a bloquear las comunicaciones desde Viguí, en Veraguas, hasta Horconcitos, en Chiriquí, basándose en medidas de seguridad, incomunicando a la población ngäbe y buglé del resto del país. Esta acción llevó a miembros de la sociedad civil panameña a acusar al gobierno de violar las garantías constitucionales de libertad de pensamiento y expresión. ${ }^{16}$

Ante estos hechos, se promulgan dos leyes que tienen importancia con respecto a la minería cuprífera en la Comarca Ngäbe-Buglé y el proyecto que había comenzado en la década de 1970. En primer lugar, está la Ley 11 del 26 de marzo de 2012, "Que establece un régimen especial para la protección de los recursos minerales, hídricos y ambientales en la comarca Ngäbe-Buglé”, y que en su artículo $3^{\circ}$ dice: "Se prohíbe el otorgamiento de concesiones para la exploración, explotación y extracción de minería metálica, no metálica y sus derivados en la comarca Ngäbe-Buglé, sus áreas anexas y las comunidades ngäbe-buglé adyacentes a estas, por cualquiera persona natural o jurídica de carácter público o privado, nacional o extranjera."

El artículo $4^{\circ}$ señala: "Se cancelan todas las concesiones otorgadas a personas naturales o jurídicas, nacionales o extranjeras, para la exploración

${ }^{15}$ Entrevista al obispo José Luis Lacunza/Entrevistadora: Flor Bocharel. Lacunza cuenta lo que pasó. La Prensa, 6 de febrero de 2012.

${ }^{16}$ Gobierno violó derechos. La Prensa, 6 de febrero de 2012.

\section{()(1) $(9$}


y explotación de los recursos minerales de la comarca Ngäbe-Buglé y áreas anexas."

Mientras que en su artículo 16 se establece: "La presente Ley deroga la Ley 41 de 1 de agosto de 1975 y el artículo 48 de la Ley 10 de 7 de marzo de 1997."

Dado que el conflicto con los grupos ngäbes y buglés tenía su origen en la oposición de estos a la explotación hidroeléctrica que el gobierno realizaba en ese momento y a la explotación minera que tenía proyectado hacer en su comarca, para calmar los ánimos, el gobierno decidió ceder totalmente en el segundo punto, con la esperanza de que no se interfiriera con el proyecto hidroeléctrico. Se cancelaba así la concesión del yacimiento de cobre en Cerro Colorado otorgada por el Estado a la CoDEmIN en 1975 y se eliminaba el acuerdo logrado por el Estado al crearse la Comarca en 1997 por el cual la exploración y explotación de los recursos naturales y minerales de la Comarca "podrán llevarse a cabo en ejecución de los planes y proyectos de desarrollo industrial, agropecuario, turístico, minero y energético, vial y de comunicación u otros, que beneficien al país de acuerdo con lo dispuesto en legislación nacional".

Previendo que con esta ley se evitarían posibles disturbios, como los sucedidos en 2010, el año anterior y meses antes, al haber separado los intereses de los grupos ngäbe y buglé de los del resto del país, el gobierno promulgó la Ley 13 del 3 de abril del 2012, "Que restablece la vigencia de artículos del código de recursos minerales y de otras disposiciones", que eliminaría la restricción a la compañía coreana interesada en desarrollar la minería en el territorio panameño al establecer en su artículo $4^{\circ}$ que "No podrán obtener concesiones mineras, ni ejercerlas o disfrutarlas, ninguno de los que a continuación se mencionan: 1. Los gobiernos o estados extranjeros ni ninguna entidad o institución oficial o semioficial extranjera."

Debe recalcarse que al comienzo de 2012, periódicos en Panamá reportaban que el consorcio de empresas liderado por la firma Korea Resources Corporation (KOREs), compañía surcoreana con participación estatal, había recibido la aprobación por parte de la Autoridad Nacional del Ambiente (AnAm) de Panamá para el desarrollo de una mina de cobre en la provincia de Colón en el primer semestre del año, con la proyección de comenzar a extraer los concentrados de cobre a partir de 2016. ${ }^{17}$

${ }^{17}$ Consorcio de Corea del Sur desarrollará mina de cobre en Panamá. Panamá América, 6 de enero de 2012. El sitio de CentralAmericaData.com (10 de enero del 2012) también informaba la compra del $20 \%$ de Minera Panamá por el consorcio surcoreano. Recuperado de http://www.centralamericadata.com/en/article/home/Inmet_Sells_20_of_ Cobre_Panama_to_Korean_Holding_Company

\section{()ㅜ(1) $\$$}


No obstante, la legalidad del otorgamiento de esta concesión se veía afectada desde la derogación de la Ley 8 de 2011, y no tendría validez jurídica hasta la aprobación de la Ley 13 de 2012, que finalmente permite a las compañías multinacionales con participación estatal extranjera beneficiarse de las concesiones mineras en suelo panameño, dejando abierta la controversia de si el Estado legisla a favor de los intereses de estas compañías o de sus ciudadanos, sobre todo por los conflictos que sucedieron hasta la promulgación de la ley.

Al haber sido los grupos ngäbe y buglé los principales opositores a las intenciones gubernamentales de explotación minera en su comarca, un punto que llama la atención de esta ley es que, a diferencia de la aprobada en 2011, el gobierno central sólo se comprometía a otorgar los beneficios de los proyectos mineros a los municipios, sin mencionar a las comarcas.

Artículo 20. Se restablece la vigencia del artículo 20 de la Ley 3 de 1988, así:

Artículo 20. A los municipios donde se realicen actividades de exploración y explotación minera por razón de una concesión, les corresponderá el 20\% de los beneficios que, de acuerdo con el Código de Recursos Minerales, debe percibir el Estado de tales actividades. Dicho ingreso será recaudado directamente por estos municipios, con base en los cálculos que prepare la Dirección Nacional de Recursos Minerales del Ministerio de Comercio e Industrias, y será dirigido únicamente a programas de desarrollo en áreas de educación, salud y proyectos de inversión socioambiental requeridos por las comunidades.

Del porcentaje establecido en este artículo, corresponderá el 5\% a los corregimientos colindantes con los municipios en un radio de 35 kilómetros de las concesiones donde se realicen actividades de exploración y explotación minera por razón de una concesión.

\section{ANÁLISIS DE LA RELACIÓN GOBERNABILIDAD DEMOCRÁTICA Y GOBERNANZA APLICADA A LA MINERÍA CUPRÍFERA EN CERRO COLORADO}

Al comparar la gobernabilidad democrática y la gobernanza en los tres periodos de estudio, se parte de que en el primero (1946-1968) la minería cuprífera no influía en esta zona: en primer lugar, se desconocía aún el valor de los yacimientos mineros, y el Código de Recursos Minerales de 1963 fue aprobado cinco años antes de terminar el periodo. Esto explica que las relaciones entre

\section{(1)(1) $\$$}


el Estado, los campesinos y los indígenas se vincularon en torno a la posesión de la tierra de cultivo, razón por la que las alianzas estratégicas se dieron entre los actores agropecuarios, entre los que se incluyen los enclaves bananeros y cafetaleros y los terratenientes de la región y el Estado por un lado, y por el otro los campesinos pobres y los indígenas, cuyo valor estaba en su mano de obra, que era aprovechada por el primer grupo.

Sin embargo, existe un cambio en la primera mitad del segundo periodo (década de 1970), cuando se intenta integrar las diferentes regiones del país, rompiendo así el modelo tradicional vigente que hasta entonces se había enfocado en la zona de tránsito, y se propuso aprovechar la exportación de minerales, convirtiendo el cobre de Cerro Colorado en uno de los pilares del nuevo proyecto nacional, que facilitaría el desarrollo y la independencia de otras regiones frente a la zona de tránsito, tales como la agropecuaria (Herrera, 2003, pp. 133-143). Se puede encontrar una doble alianza entre el Estado $y$ las empresas mineras multinacionales para explotar el proyecto minero, $y$ entre el Estado y las comunidades ngäbes y buglés para la creación de la comarca. Como muestra de gobernanza están la Ley que crea la institución concesionaria del yacimiento de cobre-CODEMIN-en 1975 y la negociación del anteproyecto para la creación de la comarca. Visto desde un plano económico, los grupos agropecuarios pudieron también unirse a las fuerzas emergentes al final de la década de 1970 contrarias al gobierno, ${ }^{18}$ temerosos de perder territorios y obreros no calificados para el trabajo a bajo costo en sus fincas, en caso de llegar a concretarse el proyecto minero y la creación de la comarca.

Con los cambios internacionales y nacionales, a partir de la mitad del segundo periodo (década de 1980) se regresa a una situación de alianzas similar a la del primer periodo, al no existir la determinación de cumplir los proyectos de explotación minera y de creación de la comarca ngäbe-buglé (o guaymí, como eran conocidos entonces), que destruyen la meta final de integración regional y de desarrollo y fortalecimiento institucional.

Si en la década de 1980 el clima económico internacional movió al Estado a negociar la entrada en una etapa de cuido y mantenimiento, en la de 1990 lo condujo a un reinicio de negociaciones. Sin embargo, ¿cómo se explica que habiéndose logrado uno de los objetivos a comienzo del tercer periodo -la creación de la comarca en 1997- no se lograse el segundo? A diferencia

${ }^{18}$ Gjording (1991, pp. 31-35) se refiere a cómo las fuerzas de la oligarquía no lograron unirse y formar una oposición al proyecto minero, y es por eso que apoyaron las demandas salariales de los maestros, politizando este movimiento, acusando al gobierno militar de comunista.

\section{()(1) $\$$}


del segundo periodo, la explotación minera perdió en el tercero la visión de ser un instrumento estatal que serviría como motor de desarrollo e integración nacional de todas las regiones del país para únicamente ser visto como una fuente de recursos para los proyectos estatales que, dada la peculiaridad del sistema político panameño -aunado al sistema neoliberal que promueve una reducción de la participación económica del Estado a favor de las empresas privadas-, es percibido por el ciudadano común como una fuente de ingresos para los gobernantes, disminuyendo así la eficacia y eficiencia de la gobernabilidad. Por otra parte, el deseo por cambiar el Código de Recursos Minerales en el quinquenio de 2009 a 2014 para ajustarlo a la medida de un futuro concesionario sólo creó tensión en la población, pues hizo evidente cómo con este proceso se perdía la legitimidad necesaria para una buena gobernabilidad, con la agravante para las comunidades ngäbes y bugles, que temieron que sin legitimidad se perderían los avances logrados en los 40 años anteriores. Esto explica que el gobierno, para calmar las tensiones, promulgara la Ley 11 de 2012 que excluye su región de las decisiones mineras del resto del país, esto significó un retroceso de 40 años al eliminar a la compañía estatal concesionaria del yacimiento minero, perdiéndose una oportunidad alternativa de desarrollo tanto para el Estado como para la Comarca, ya que la prisa por aprobar una ley cuyos beneficios fueron asociados al gobierno y sus allegados, provocó el rechazo de la población.

\section{COMPONENTES DE LA GOBERNANZA AMBIENTAL APLICABLES A LA MINERÍA CUPRÍFERA}

Por otro lado, no es posible que en el caso de la minería cuprífera el Estado otorgue concesiones especiales contraviniendo el Código de Recursos Minerales, violando el imperio de la ley necesario para una buena gobernabilidad y gobernanza, ya que los requisitos para ser concesionario minero, metálico y no metálico, para la exploración y explotación están claramente documentados en el código: capacidad técnica, los impuestos, publicaciones y otros, y los mismos son aplicados sin distinción. ${ }^{19}$

19 Luque, V. (11 de diciembre de 2013). Entrevista con el profesor en derecho minero Lic. Virgilio Luque/Entrevistador: Jorge Luis Roquebert León. Universidad de Panamá, Panamá. No obstante, sí podrían ocurrir fallas, si bien no por parte del Estado central, sino por los municipios en el manejo de los recursos minerales, dado que la Ley 55 de 1973, en sus artículos 33,37 y 38 , faculta a los alcaldes el otorgamiento de permisos a personas naturales para la

\section{()ㅜ(1) $\$$}


Además, dentro de las disposiciones que Panamá tiene relacionadas con la gobernanza ambiental, desde inicios de 1991 la Dirección Nacional de Recursos Minerales exige la entrega de un informe de impacto ambiental como requisito para ser concesionarios (Resuelto 3791 de 1991, de Recursos Minerales), siendo la primera institución en hacerlo, incluso antes de que la Autoridad Nacional del Medio Ambiente lo solicitara, a finales del mismo año.

Se puede asumir que la institución, conociendo que en Panamá el tema minero es conflictivo, aún más si se presenta unido al tema ambiental, buscó un mecanismo para responder a las demandas de la población de un ambiente sano. ${ }^{20}$

\section{PERSPECTIVAS DE LA GOBERNABILIDAD TERRITORIAL EN EL CASO DE ESTUDIO}

Si la gobernabilidad territorial ideal se alcanza cuando ocurre un ganar-ganar de los actores en juego, se puede asumir que el tema de la minería fue uno de los factores que movió a comenzar en el segundo periodo el intercambio del reconocimiento de la comarca por parte del Estado a cambio de la aceptación de la explotación minera de Cerro Colorado por los habitantes del territorio. Su importancia es notoria cuando dos años después de decidirse posponer el proyecto, el siguiente gobierno no se sintió comprometido a seguir dicho proceso, y no se aprobaron las negociaciones de creación de la comarca en 1983, alterando incluso el proyecto original (Herrera, 2012, pp. 54-55).

En el tercer periodo la situación ha variado y las perspectivas de los actores son diferentes. El Estado, teniendo una visión diferente de los usufructos que representaría el cobre para su administración, se adelanta y

extracción de material de ríos para la construcción de sus viviendas -especificando tipos de minerales y cantidad a ser extraída- además de requisitos como el de ser sin fines de lucro, extraídos por el interesado, y con un costo no mayor a B/. 5,000.00 (existe una paridad entre el balboa panameño y el dolar estadunidense; es decir, las dos monedas han sido de curso legal en Panamá). Además, el artículo 33 de la Ley 109 de 1973 también reafirma que los alcaldes dan permisos para la extracción de pequeñas cantidades de minerales no metálicos. Aunque no entra en los límites de la investigación, por no tratarse del metal cuprífero, se debe conocer sobre la falla existente en la institución encargada de darle seguimiento a las concesiones municipales, incluso dentro de la comarca, porque la Ley 11 de 2012 no prohibió las concesiones de piedra, tosca, gravilla y arena para proyectos sociales en beneficio de esta.

${ }^{20}$ El profesor Luque explicó también que al igual que cualquier proyecto nacional, para explotar los yacimientos debe seguirse también lo acordado por la Ley de Ambiente, que está en el Decreto Ejecutivo 123 de 2011.

\section{()(1) $(3$}


aprueba la comarca, si bien, al contrario del segundo periodo, cuando las negociaciones de exploración y explotación del recurso cuprífero estaban en marcha, en este periodo no habían aún comenzado. A las poblaciones ngäbe y buglé el tiempo les ha permitido ganar más conciencia de su fortaleza como grupo, y la creación de la comarca es vista como un derecho que poseen, y la explotación minera, de ser posible, deberá estar acompañada de nuevas negociaciones y concesiones. Al seguirse una política de gobernabilidad vertical, poco importó a los grupos ngäbes y buglés que el Estado ofreciera mejores ganancias económicas, como el aumento del porcentaje que recibirían las comunidades donde se realizaran los proyectos mineros, sino que decidieron defender su territorio, provocando incluso una crisis de gobernabilidad cuando el gobierno sobrepasó los límites de la fuerza legalmente permitida.

\section{CONTRIBUCIÓN AL DESARROLLO HUMANO SOSTENIBLE}

Vargas Hernández (2004), al desarrollar el concepto del capital social, explica:

La globalización de los fenómenos económicos, políticos y sociales contemporáneos requiere ser replanteada desde una perspectiva humanista con el fin de establecer equilibrios y balances entre el crecimiento económico, el desarrollo socio-político, la democracia y el bienestar de la sociedad. La globalización está aquí para quedarse, pero el neoliberalismo puede ser parcialmente manejado. Las políticas neoliberales que han llevado a una creciente desigualdad necesitan una reversa urgente porque amenazan la democracia global y complican la crisis del medioambiente (p. 42).

Así como menciona que la globalización ha llegado para quedarse, también los proyectos mineros estarán vigentes en Panamá mientras existan recursos rentables. Las políticas neoliberales establecidas, que no han fijado su eje de desarrollo en la población, deben ser llevadas a un punto de equilibrio que permita a la población tener un acceso a la igualdad de oportunidades y al desarrollo social, para lograr una gobernabilidad democrática ideal.

Se ha expuesto cómo la visión del recurso cuprífero en Cerro Colorado ha variado en las últimas cuatro décadas: desde ser considerado en la primera parte del segundo periodo de estudio (década de 1970) como la punta de lanza a ser utilizada por el Estado para obtener los ingresos para desarrollar

\section{(이요 $\$$}


sus proyectos de gobierno y depender menos de los dictámenes de Estados Unidos, que en esta década controlaba directamente la zona de tránsito, disminuyéndose el dominio que ejercía en la economía panameña al diversificarse, hasta ser visto en la actualidad como una fuente de recursos para los propietarios, de los que el Estado se excluye como tal, haciendo concesiones a personas allegadas a los gobiernos en turno que pactan con empresas mineras multinacionales.

Si bien a finales de la década de 1980 se procuró calmar los ánimos de la población otorgando una parte mayor de las regalías obtenidas, el trabajo no se completará hasta que los municipios logren manejar directamente este ingreso, creando proyectos propios para su comunidad. Mientras no se logre una visión donde la minería vuelva a ser un medio de desarrollo que esté al servicio de todos, buscándose siempre un consenso general para el desarrollo de otras áreas en rezago, los proyectos encontrarán oposición por parte de la población panameña en general. ${ }^{21}$

\section{CONCLUSIONES}

Un trabajo que tenga como propuesta estudiar la relación histórica entre el Estado panameño y el grupo ngäbe entre 1946 y 2014, sin tratar el yacimiento de cobre en Cerro Colorado estaría incompleto.

El manejo del tema minero es un claro ejemplo de cómo ha cambiado la relación entre el Estado y el grupo ngäbe; durante el primer periodo de estudio estos grupos tenían escasas posibilidades de que su opinión fuera considerada. En el periodo actual, conscientes de sus derechos, han ejercido

${ }^{21}$ Millones de la minería no llegan aún a Donoso. La Prensa, 18 de febrero de 2014; Muere figura del fideicomiso en Donoso. La Prensa, 9 de diciembre de 2014. El municipio de Donoso es un claro ejemplo del beneficio económico que recibirían comunidades apartadas al compensarles las regalías acordadas por la Ley 13 del 2012. Este municipio de 14000 personas, con ingresos anuales de B/. 400, recibía del Estado para su operación B/. 200000. Al igual que el distrito vecino de Chagres, no cuenta con agua potable, vertedero para la basura, ni carretera que los comunique por la costa caribeña a la ciudad de Colón. En octubre del 2012 la empresa Minera Panamá pagó en concepto de impuestos municipales B/. 3.6 millones, que no se pueden utilizar por la decisión de depositarlos en el banco y crear un fideicomiso. En diciembre de 2014, se anunció un nuevo aporte de B/. 1.5 millones en pago de impuestos, y el nuevo alcalde de Donoso señaló que no sería utilizada la figura de fideicomiso en donde el dinero queda "inerte", sino que se atenderían obras necesarias para la comunidad. También declaró que se llegó a un acuerdo con la empresa minera para entregar el nuevo pago en enero del presente de B/. 4.5 millones al municipio directamente.

\section{()(1) $\$$}


medidas de presión y han logrado sentar en la mesa de diálogo al gobierno central para una nueva negociación o incluso han logrado en los últimos cuatro años la paralización parcial de los proyectos en su comarca.

Es preocupante el cambio de la visión del Estado panameño frente a sus recursos naturales. En el segundo periodo, en la década de 1970, el gobierno central encontró la manera de negociar más equitativamente con las compañías mineras internacionales, sabiéndose en desventaja tecnológica y económica, pero dueño de la concesión, y visualizó la explotación de los recursos minerales como una fuente de desarrollo para otras áreas del país en busca de la construcción de un Estado de bienestar; esta negociación también sirvió al grupo ngäbe como una herramienta para obtener el territorio reclamado a cambio de aceptar que el recurso natural fuera transformado en el capital necesario para cumplir los proyectos de inversión del Estado. Sin embargo, este proyecto no se concretó y tampoco las siguientes metas. En el tercer periodo de estudio, a partir de 1990, se pretendía imponer el desarrollo de los proyectos para la explotación de los recursos naturales, ya fueran hidroeléctricos o minerales, chocando con la oposición de la población local que veía cómo proyectos similares en áreas vecinas habían beneficiado mayormente a grupos foráneos, que convirtieron los recursos naturales panameños en capital extranjero.

Durante la década de 1980 se suspendieron los trabajos de explotación de los yacimientos cupríferos, decisión que fue adoptada tras las directrices impuestas por los organismos financieros internacionales vinculados al proyecto, que perseguían mantener una participación mínima del Estado nacional en la economía. Esta paralización temporal configura un ejemplo claro de la corta visión estatal y de la impericia del gobierno por aceptar como argumento válido y suficiente la caída del precio del mineral registrada en 1981. La situación varió y se superó completamente seis años más tarde, lo que significa que, de haberse continuado con el desarrollo del proyecto, para 1987, cuando el cobre volvió a estabilizar su precio en el mercado internacional, los accionistas de esta compañía, 50\% estatal, hubieran obtenido una importante utilidad en vez de pérdidas.

De no haberse interrumpido por más de tres lustros los trabajos de explotación de los yacimientos de Cerro Colorado, el plan de creación de la comarca hubiera avanzado, y se habrían anejado otros logros, expresados en la integración de nueva tecnología y de capital humano nacional, que mitigaría con mucho la repartición desigual de las ganancias que ha afectado a toda la población del país. En este sentido es necesario abordar un cambio de visión

\section{()(1) $(3$}


del Estado acerca de las comarcas para que no sean tratadas como un territorio para el olvido de poblaciones marginadas, sino para el desarrollo de los grupos con base en su cultura. Que el Estado recuerde las necesidades de la comarca sólo en función de los beneficios que pueda obtener de ella, como los mineros o los hidroeléctricos, provoca la suspicacia de sus habitantes.

Si estos proyectos dejaran una proporción de las regalías en la comarca y sirvieran para desarrollar otros sectores económicos descuidados se lograría una mayor aceptación por parte de la ciudadanía. La experiencia en otras comunidades del país, también pobres y con necesidades fundamentales por resolver, en donde no existe una planificación de cómo utilizar los nuevos ingresos por concepto de los impuestos municipales, invita a una planificación por parte de las instituciones responsables de ello para terminar con la práctica de beneficiar al gobierno central y para convertir el recurso de la comunidad en un capital propio que sirva en obras como escuelas, carreteras y centros de salud inexistentes, en beneficio de los residentes.

La explotación de yacimientos debe continuar siendo vista como un recurso importante para el desarrollo y el fortalecimiento de la economía del país sin menoscabo de las consideraciones culturales e históricas que rodean este tipo de proyectos y sin descartar tampoco el tema de los riesgos ambientales que apareja toda actividad humana, en este caso la minería, que son superables con el cumplimiento cabal de las normas vigentes para este sector. Por la evolución del papel del Estado en el tema minero y energético - de ser accionista a nuevamente sólo otorgar concesiones-y la nueva visión ambiental en estos 40 años, se debe buscar la creación de una institución que coordine los tres temas, ambiental, minero y energético, y no esperar a la creación de tres instituciones que los coordinen cada una individualmente ya que eso sólo debilita la capacidad de respuesta del Estado a sus ciudadanos.

Los yacimientos cupríferos en Cerro Colorado muestran el cambio de la relación entre el Estado y el grupo ngäbe, ya que un recurso natural que era de todos los panameños a mediados del siglo xx, en la actualidad tiene la peculiaridad de estar en la Comarca Ngäbe-Buglé, creada a finales de siglo. Este hecho debe servir para detener la exclusión y la usurpación de riquezas existente desde el choque de culturas hace 500 años, y convertirse en motor para que pueblos históricamente explotados, participantes de nuestra economía capitalista nacional en su escala más baja, tengan las mismas oportunidades que el resto de sus conciudadanos. La negociación es inminente para construir la gobernabilidad democrática participativa que los panameños anhelamos.

\section{()(1) $\$$}




\section{LISTA DE REFERENCIAS}

Cuadra, Á. (9 de septiembre de 2013). Chile a cuarenta años: crónica de un golpe de Estado. Recuperado de http://utopialapalabra.blogspot.com/2013/09/chile-cuaren ta-anos-cronica-de-un-golpe.html

Gandásegui, M. A. (2008). La concentración del poder económico. En M. A. Gandásegui, Las clases sociales en Panamá (pp. 113-182). Panamá: celA.

Gjording, C. N. (1991). The Guaymí indians and mining multinationals in Panama. Washington: Smithsonian Institution.

Herrera Jurado, L. (2003). Población, ambiente y salud. En L. Herrera Jurado, El país que somos, 30 años de Geografía Humana en Panamá (pp. 133-143). Panamá: Instituto de Estudios Nacionales/Universidad de Panamá.

Herrera, F. (2012). La evolución de las demandas indígenas sobre la tierra y las respuestas del Estado en Panamá. QuAderns-E, 17, 44-59.

Manduley, J. (2014). La política económica de Omar Torrijos. De la plataforma de servicios al Estado delincuencial. Tareas, 97-121.

Manfredo, F. (1976). Epílogo de la guerra fiscal del banano. Revista Lotería, 3-17.

Mena García, C. (2011). El oro del Darién. Entradas y cabalgadas en la conquista de Tierra Firme (1509-1525). Sevilla: Fundación Pública Andaluza Centro de Estudios Andaluces.

Soler, R. (2008). Panamá, nación y oligarquía. En M. A. Gandásegui, Las clases sociales en Panamá (pp. 79-111). Panamá: cela.

Vargas Hernández, J. G. (2004). Formación de capital social para fortalecer la institucionalización de la gobernabilidad. Recuperado de http://www.ull.es/publicacio nes/latina/20040657vargas.htm

Ventura, D. (11 de septiembre de 2013). Por qué el golpe de Estado en Chile es tan emblemático. Recuperado de: http://www.bbc.co.uk/mundo/noticias/2013/09/130906_ chile_11_septiembre_golpe_emblematico.shtml

\section{OTRAS FUENTES}

\section{Bibliografía}

Codelco (s. a.). Historia de Codelco. Recuperado de http://www.codelco.com/histo ria/prontus_codelco/2011-02-25/103555.html

Duarte, C. (6 de enero de 2012). Gobernabilidad minera: cronología legislativa del subsuelo en Colombia. Recuperado del sitio web del Centro de Pensamiento RAIZAL:

\section{()ㅜ(1) $\$$}


http://gobernabilidadminera.files.wordpress.com/2012/01/gobernabilidad-miner a-cronologicc81as-legislativas-del-subsuelo-en-colombia.pdf

Geología de Azuero (s. a.). Apuntes sobre Cerro Colorado (parte I). Recuperado de http://geologia.azueropanama.org/2011/02/cerro-colorado/

\section{Periódicos}

La Prensa.

Panamá América.

\section{Leyes}

Ley 55 de 1973. Por la cual se regula la administración, fiscalización y cobro de varios tributos municipales.

Ley 41 de 1975. Por la cual se crea la Corporación de Desarrollo Minero Cerro Colorado. Ley 8 de 1987. Por la cual se regulan las actividades relacionadas con los hidrocarburos. Ley 3 de 1988. Por la cual se reforma el Código de Recursos Minerales.

Ley 10 de 1997. Que crea la comarca y regula la relación de esta y el Estado panameño. Ley 8 de 2011. Que reforma el Código de Recursos Minerales y dicta otras disposiciones relacionadas con la actividad minera.

Ley 11 de 2012. Que establece un régimen especial para la protección de los recursos minerales, hídricos y ambientales en la Comarca Ngäbe-Buglé.

Ley 13 de 2012. Que restablece la vigencia de artículos del código de recursos minerales y de otras disposiciones. 